\title{
Characteristic of FINDRISC Score and Association with Diabetes Development in 6-Year Follow-Up in PURE Poland Cohort Study
}

\author{
Katarzyna Zatońska' \\ Alicja Basiak-Rasała (D) ${ }^{\prime}$ \\ Katarzyna Połtyn-Zaradna' \\ Dorota Różańska ${ }^{2}$ \\ Maciej Karczewski \\ Maria Wołyniec' \\ Andrzej Szuba ${ }^{4}$ \\ 'Department of Social Medicine, \\ Wroclaw Medical University, Wrocław, \\ Poland; ${ }^{2}$ Department of Dietetics, \\ Wroclaw Medical University, Wrocław, \\ Poland; ${ }^{3}$ The Faculty of Environmental \\ Engineering and Geodesy, Department of \\ Mathematics, Wroclaw University of \\ Environmental and Life Sciences, \\ Wrocław, Poland; ${ }^{4}$ Department of \\ Angiology, Hypertension and \\ Diabetology, Wroclaw Medical \\ University, Wroclaw, Poland
}

Purpose: The aim of the study was to assess if FINDRISC score was associated with diabetes development after 6 years of observation.

Methods: Polish cohort is a part of global PURE study. Hereby analysis presents data from baseline (2007-2010) and 6-year follow-up (2013-2016) and was conducted on 1090 participants (702 women) from urban and rural areas in Lower Silesia region (Poland) without diabetes at the baseline and with complete data throughout course of the study.

Results: At the baseline, women had significantly higher FINDRISC score than men (10.43 vs 8.91; $\mathrm{p}=0.000)$ and participants from rural areas had higher score than from urban areas (10.97 vs 9.33; $\mathrm{p}=0.000$ ). At the baseline, $25.87 \%$ of the participants had low risk of diabetes according to FINDRISC score, $38.90 \%$ had slightly elevated risk, $16.79 \%$ moderate risk, $16.42 \%$ high risk and $2.02 \%$ very high risk. Participants, who were healthy at baseline, but developed diabetes after 6 years of observation had significantly higher FINDRISC, than those who did not (13.39 vs 9.36; $\mathrm{p}=0.000$ ). In 6-year follow-up, diabetes was diagnosed in $2.8 \%$ of participants, who were ascertained to "low risk" according to FINDRISC score in baseline; in 9.9\% of participants of "slightly elevated risk", $17.5 \%$ of participants of "moderate risk", $26.8 \%$ in participants of "high risk" and $50.0 \%$ of participants of "very high risk".

Conclusions: Results of PURE Poland cohort study indicates that higher FINDRISC score at the baseline was associated with higher risk of diabetes development during 6 years of observation.

Keywords: diabetes, preventive medicine, epidemiology

\section{Plain Language Summary}

Worldwide prevalence of diabetes is rapidly increasing. In order to counteract diabetes epidemic, it is essential to identify and target population at higher risk of diabetes development. The FINDRISC score proved to be reliable and an easy tool to identify population at high risk of diabetes development in European countries. Results of PURE Poland cohort study indicate that higher FINDRISC score was associated with higher risk of diabetes development. Almost one fifth of participants of "moderate risk", almost one third of participants of "high risk" and half of participants of "very high risk" will develop diabetes in 6 years. It shows that even participants with moderate risk according to FINDRISC should be systematically tested for blood glucose.

Correspondence: Alicja Basiak-Rasała Department of Social Medicine, Wrocław Medical University, O. Bujwida 44 Street, Wrocław, 50-345, Poland $\mathrm{Tel} / \mathrm{Fax}+48713282145$

Email alicja.basiak-rasala@umed.wroc.pl

\section{Introduction}

Worldwide prevalence of diabetes is rapidly increasing. According to International Diabetes Federation (IDF), diabetes affects $463 \mathrm{mln}$ people $(9.3 \%$ of current global 
population) and impaired glucose tolerance affects a further $373 \mathrm{mln}$ people (7,5\% of population). If current trends remain steady, the number of people affected by diabetes may reach $700 \mathrm{mln}$ in $2045 .{ }^{1} 8 \%$ of Polish population suffers from diabetes and it is estimated that this percentage will increase to $11 \%$ in $2040 .^{2}$ Socioeconomic impact of diabetes is alarming. In Europe, $8.3 \%$ of total health costs is attributed to the treatment of diabetes and its complications. ${ }^{1}$ A total of $11.3 \%$ of global deaths in adult population can be attributed to diabetes. ${ }^{1}$ It is estimated that diabetes increases the risk of cardiovascular events and overall mortality 2-4-fold in comparison to healthy controls. ${ }^{3}$ Hyperglycemia causes endothelial dysfunction, which contributes to macro- and microvascular complications of diabetes. ${ }^{4}$

In order to counteract diabetes epidemic, it is essential to identify and target population at higher risk of diabetes development. Recent studies indicate that introducing lifestyle intervention in patients with prediabetes results in delaying or preventing the onset of full-symptomatic diabetes. ${ }^{5}$ Improving physical activity, diet quality and reducing excessive body mass resulted in 58\% reduction of diabetes risk in participants with impaired glucose tolerance. ${ }^{6}$ According to IDF, $50 \%$ of diabetes cases (mostly type 2 diabetes) are undiagnosed, which brings urgent need to improve screening. To effectively identify targeted population, tools used in the screening should be noninvasive, easy to perform and reliable. Finnish Diabetes Risk Score was designed and validated by Lindström et al in the FINDRISC study cohort. ${ }^{7}$ The FINDRISC score proved to be reliable and an easy tool to identify population at high risk of diabetes development. According to Kengne et $\mathrm{al}^{8}$ who performed a validation of diabetes risk scores in the EPIC-InterAct study population, concluded that non-invasive risk scores including FINDRISK have acceptable to good discriminatory power and can be reasonably recommended to identify people at high risk of diabetes. Although the FINDRISC score has been originally validated to assess the 10-year risk of development of diabetes, ${ }^{7}$ it has been previously used in 7-year or 9-year assessments of diabetes risk. ${ }^{9-11}$ The prevalence of diabetes increased rapidly over 6 years of observation of the PURE Poland cohort study, ${ }^{12}$ hence the decision to check if the FINDRISC score assessed at the baseline predicted the diabetes development in this study period.

\section{Objectives}

The Finnish Diabetes Risk (FINDRISC) Score was calculated in participants without diabetes at the baseline to check whether higher FINDRISC is associated with diabetes development after 6 years of observation. Secondary aim was to determine differences in FINDRISC score in the view of chosen sociodemographic characteristics.

\section{Methods}

Prospective Urban and Rural Epidemiology Study (PURE) comprises more than 21 countries from different socioeconomic backgrounds. Poland was initially included as one of the middle income countries. The cohort consisted of participants from both urban (Wrocław) and rural (Wrocław's whereabouts) place of residence. All participants were examined following the same global protocol of PURE study. ${ }^{13}$ Study design included questionnaire, blood collection, blood pressure measurements, spirometry, anthropometric measurements. Blood glucose level was determined in the fasting state. Baseline data was collected between 2007 and 2010. Follow-up data is collected every two years. Data for 6-year follow-up was collected between 2013 and 2016. Hereby analysis presents data from baseline and 6-year follow-up. Initially, 2036 participants were recruited at the baseline. 222 participants with diabetes diagnosed at the baseline were excluded from this analysis. Further 478 participants were removed from the analysis due to lack of data in 6-year follow-up (deaths, refusals, lack of glucose measurement and incomplete interview). The analysis was performed on remaining 1090 participants, without diabetes at the baseline and with complete data throughout course of the study. According to current consensus, ${ }^{14}$ participants were considered normoglycemic when their fasting plasma glucose was between 70-99 mg/dL (3.9$5.5 \mathrm{mmol} / \mathrm{L}$ ) and they had no diabetes diagnosis. Participants were ascertained to IFG group, when their fasting plasma glucose was between $100 \mathrm{mg} / \mathrm{dL}(5.6$ $\mathrm{mmol} / \mathrm{L})$ and $125 \mathrm{mg} / \mathrm{dL}(6.9 \mathrm{mmol} / \mathrm{L})$. Participants were considered diabetic when their fasting plasma glucose was $\geq 126 \mathrm{mg} / \mathrm{dL}$ ( $7.0 \mathrm{mmol} / \mathrm{L})$, or when they reported diabetes diagnosis and taking medication.

The Finnish Diabetes Risk Score (FINDRISC) was calculated based on methodology by Lindström et al. ${ }^{7}$ The questionnaire consists of 8 questions of known diabetes risk factors. Score values were ascertained to every answer according to the key ${ }^{7}$ and overall FINDRISC was 
calculated as the sum of those values. Survey included questions regarding participant's age, Body Mass Index (BMI), waist circumference, level of physical activity, consumption of vegetables and fruit, hypertension medication, hyperglycemia and family history of diabetes. International Physical Activity Questionnaire (IPAQ) ${ }^{15}$ was used to assess the level of physical activity. We had to introduce some adjustments to calculate FINDRISC, due to availability of retrospective data in our cohort. In an original questionnaire, a query regarding frequency of consumption of vegetables and fruit had only two possible answers "every day" and "not every day". Because in PURE study nutritional data was obtained from Food Frequency Questionnaire, ${ }^{16}$ consumption of every food product was calculated to reflect the intake of grams/day. We have therefore calculated overall consumption of vegetables and fruit in grams/ day. Question about consumption of vegetables and fruit in FINDRISC reflects adherence to nutritional recommendations. Because both international ${ }^{17}$ and Polish nutritional guidelines ${ }^{18}$ state that vegetables and fruit should be consumed in amount at least $400 \mathrm{~g} / \mathrm{d}$ in order to maintain health, we decided to divide the participants in two groups: 1) those, who consume $\geq 400 \mathrm{~g}$ of vegetables and fruit per day and 2) those who consume less vegetables and fruit per day, who therefore do not meet the requirements. In case of question 7 regarding history of hyperglycemia, we had only information about participants with impaired fasting glucose (IFG). In case of IFG, participants received maximum of 5 points in this category. Regarding the last question about diabetes family history, PURE study protocol included only information about mother's, father's and siblings' diabetes history, but lacked data about other family members (FINDRISC includes also uncles/aunts/grandparents). As a result, our categorization in case of this question was dichotomous as either 1) no family history of diabetes or 2) diabetes in mother/father/sibling. Scores received in every category were later summed to overall FINDRISC. Participants could have received between 0 and 26 points in the questionnaire. Score 0-6 points reflects low risk of diabetes development; 7-11 points reflect slightly elevated risk; $12-14$ points reflect moderate risk; $15-20$ points reflect high risk and $>20$ points reflect very high risk of diabetes development. ${ }^{19}$

The analysis was performed in $\mathrm{R}$ for Windows (version 3.5.3) ${ }^{20}$ Wilcoxon rank sum test with continuity correction and Kruskal-Wallis rank sum test were used to characterize FINDRISC at the baseline (differences in average score between men/women, rural/urban place of residence and levels of education). Effect size was computed with the use of Vargha-Delaney A measure. Fisher's Exact Test with Count Data with simulated p-value was used in qualitative assessment of FINDRISC groups of risk (occurrence of diabetes in every risk group and sociodemographic characteristics in groups). Written and informed consent was obtained from all participants enrolled in the study. The study has been reviewed and accepted by the Bioethics Committee of the Wroclaw Medical University and has therefore been performed in accordance with the ethical standards laid down in an appropriate version of the 1964 Declaration of Helsinki (Positive opinion of The Bioethics Committee of the Wrocław Medical University nr KB- 443/2006).

\section{Patients and Public Involvement}

Patients or the public were not involved in the design, conduct, reporting, or dissemination plans of this research.

\section{Results}

A total of 1090 participants without diabetes at the baseline were enrolled to analysis (702 women and 388 men). At the baseline, average FINDRISC in participants without diabetes equaled 9.88. At the baseline, women had significantly higher FINDRISC score than men (10.43 vs 8.91; $\mathrm{p}=0.000$ ). There were also significant differences in FINDRISC score in participants with different level of education. Average FINDRISK score in participants with primary education was 12.02 , with secondary education 10.18, trade school education 9.91 and university education 8.92. Kruskal Wallis rank sum test showed significant differences between every education group, except between university and trade school and secondary and trade school. Effect size computed with the use of VarghaDelaney A measure showed small differences in most comparisons, but significant difference between university and primary education (average strength 0.678). Average score in participants from urban place of residence was 9.33 and from rural place of residence $10.97(\mathrm{p}=0.00)$. Sociodemographic characteristic of FINDRISC score is presented in Table 1. Considering FINDRISC variables at the baseline, the average age of the participants was 53.5 years, the average BMI was $27.5 \mathrm{~kg} / \mathrm{m}^{2}$, and average waist circumference was $90.3 \mathrm{~cm}$. $98.9 \%$ of participants declared at least 30 minutes of physical activity per day, $72.0 \%$ of participants consumed at least $400 \mathrm{~g} / \mathrm{d}$ of fruit 
Table I Sociodemographic Characteristics of FINDRISC Score

\begin{tabular}{|c|c|c|c|c|c|c|}
\hline & $\begin{array}{l}\text { FINDRISC 0-6 } \\
\mathrm{n}(\%)\end{array}$ & $\begin{array}{l}\text { FINDRISC 7-I I } \\
\text { n(\%) }\end{array}$ & $\begin{array}{l}\text { FINDRISC I2-I4 } \\
\mathrm{n}(\%)\end{array}$ & $\begin{array}{l}\text { FINDRISC I5-20 } \\
\mathrm{n}(\%)\end{array}$ & $\begin{array}{l}\text { FINDRISC >20 } \\
\mathrm{n}(\%)\end{array}$ & $\begin{array}{l}\text { FINDRISC } \\
\text { Mean }\end{array}$ \\
\hline \multicolumn{7}{|l|}{ Sex } \\
\hline Men & $126(32.5)$ & I5I (38.9) & $63(16.2)$ & $41(10.6)$ & $7(1.8)$ & 8.91 \\
\hline Women & $156(22.2)$ & 273 (38.9) & $120(17.1)$ & $138(19.7)$ & $15(2.1)$ & 10.43 \\
\hline \multicolumn{7}{|l|}{ Education } \\
\hline Primary & $14(12.1)$ & $4 \mathrm{I}(35.3)$ & $19(16.4)$ & 37 (31.9) & $5(4.3)$ & 12.02 \\
\hline Secondary & $100(23.1)$ & $173(40.0)$ & $73(16.9)$ & $77(17.8)$ & $10(2.3)$ & 10.18 \\
\hline Trade & $33(21.7)$ & $67(44.1)$ & $37(24.3)$ & $13(8.6)$ & $2(1.3)$ & 9.91 \\
\hline University & I 35 (34.7) & $143(36.8)$ & 54 (I3.9) & $52(13.4)$ & $5(1.3)$ & 8.92 \\
\hline \multicolumn{7}{|c|}{ Place of residence } \\
\hline Urban & $213(29.6)$ & $285(39.6)$ & $112(15.6)$ & $99(13.8)$ & $10(1.4)$ & 9.33 \\
\hline Rural & $69(18.6)$ & $139(37.5)$ & 71 (19.1) & $80(21.6)$ & $12(3.2)$ & 10.97 \\
\hline
\end{tabular}

and vegetables, $27.16 \%$ were treated for hypertension, $31.6 \%$ had impaired fasting glucose and $26.1 \%$ had family history of diabetes among closest relatives. The FINDRISC variables are presented in Table 2.

At the baseline, $25.87 \%$ of the participants had low risk of diabetes according to FINDRISC score, 38.90\% had slightly elevated risk, $16.79 \%$ moderate risk, $16.42 \%$ high risk and $2.02 \%$ very high risk. Women were ascertained to the groups of higher risk significantly more often than men $(p=0.0005$; effect size low $=0.15)$. At the baseline, $22.22 \%$ of women had low risk, $38.89 \%$ had slightly elevated risk, $17.09 \%$ had moderate risk, $19.66 \%$ had high risk and $2.14 \%$ had very high risk. Subsequently, $32.47 \%$ of men had low risk, $38.92 \%$ had elevated risk, $16.24 \%$ had moderate risk, $10.57 \%$ had high risk and $1.80 \%$ had very high risk. Participants with primary education were ascertained to the group of high risk significantly more often than participants with different level of education $(\mathrm{p}=0.0005)$.

At the 6-year follow-up 141 cases of diabetes were diagnosed (12.94\%). Interestingly, participants who were healthy in baseline but developed diabetes after 6 years of observation had significantly higher FINDRISC than those who remained stable. The average FINDRISC in participants who did not develop diabetes equaled 9.36 and in participants who developed diabetes equaled 13.39 $(\mathrm{p}=0.000)$. At 6-year follow-up diabetes was diagnosed in $9.97 \%$ of women $(n=70)$ and in $18.30 \%$ of men $(n=71)$.

We have also performed qualitative assessment of the FINDRISC score ascertaining the participants to groups reflecting severity of diabetes risk. Qualitative assessment of FINDRISC score reflecting differences in the $\%$ of participants with and without diabetes after 6-years of observation is presented in Figure 1. After 6 years diabetes was diagnosed in $2.8 \%$ of participants, who were ascertained to "low risk" according to FINDRISC score in baseline; in $9.9 \%$ of participants of "slightly elevated risk", $17.5 \%$ of participants of "moderate risk", $26.8 \%$ in participants of "high risk" and $50.0 \%$ of participants of "very high risk". However, it should be noted that overall number of participants in the "very high risk" category was low $(\mathrm{n}=22)$ (Table 3).

\section{Discussion}

In our study, we have assessed the risk of diabetes development by calculating the FINDRISC score in baseline population without diabetes and compared those results with occurrence of diabetes after 6 years of observation. Although the FINDRISC score has been originally validated to assess the 10-year risk of diabetes development, ${ }^{7}$ due to the high increase in diabetes prevalence in our cohort during 6 years of observation ${ }^{12}$ we decided to check if the FINDRISC score was predictive of this outcome. Our results show that the FINDRISC score was significantly higher at the baseline in those who progressed to diabetes after 6 years, than in those who remained stable. The FINDRISC score was significantly higher in women, participants living in rural areas and those with lower level of education. 


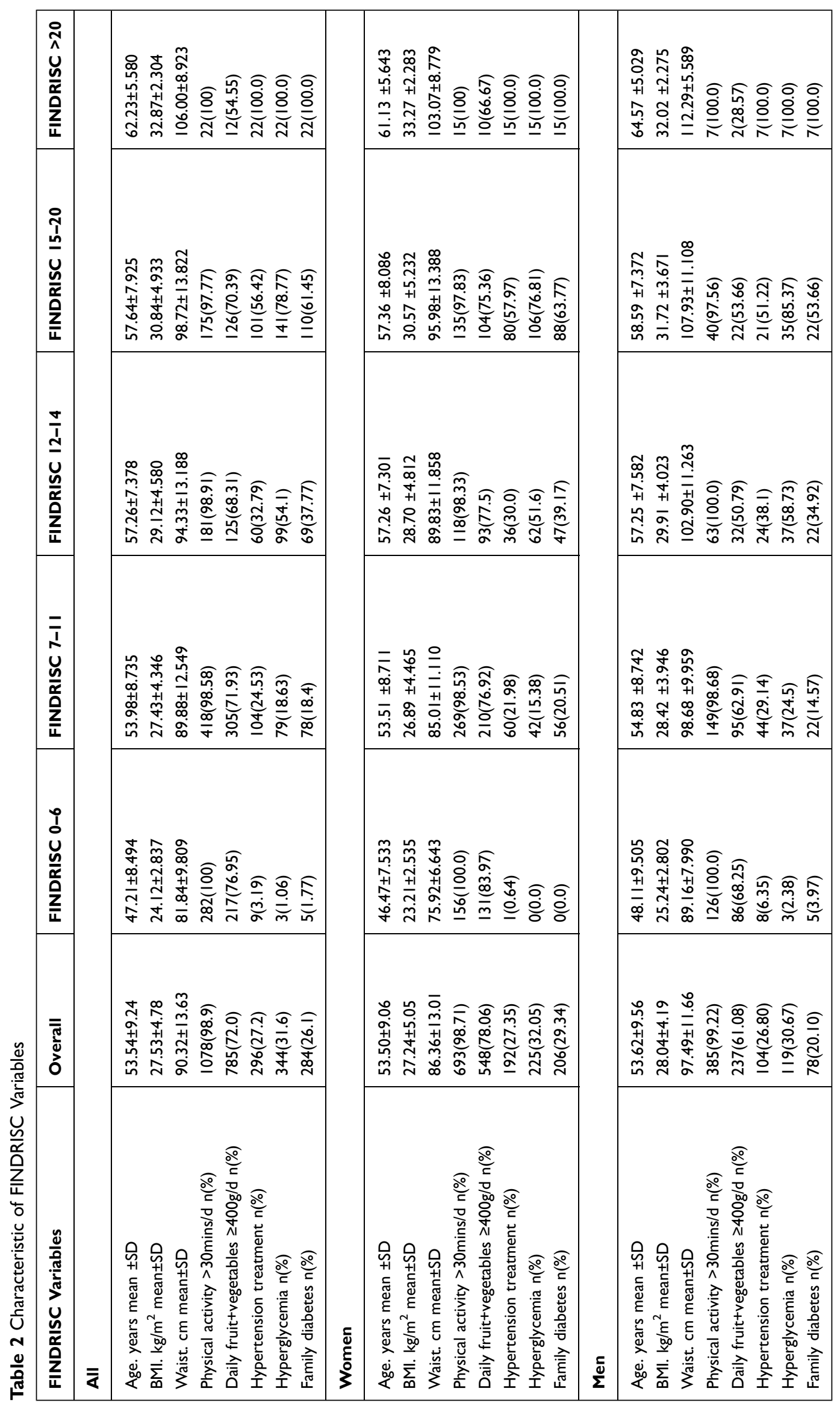




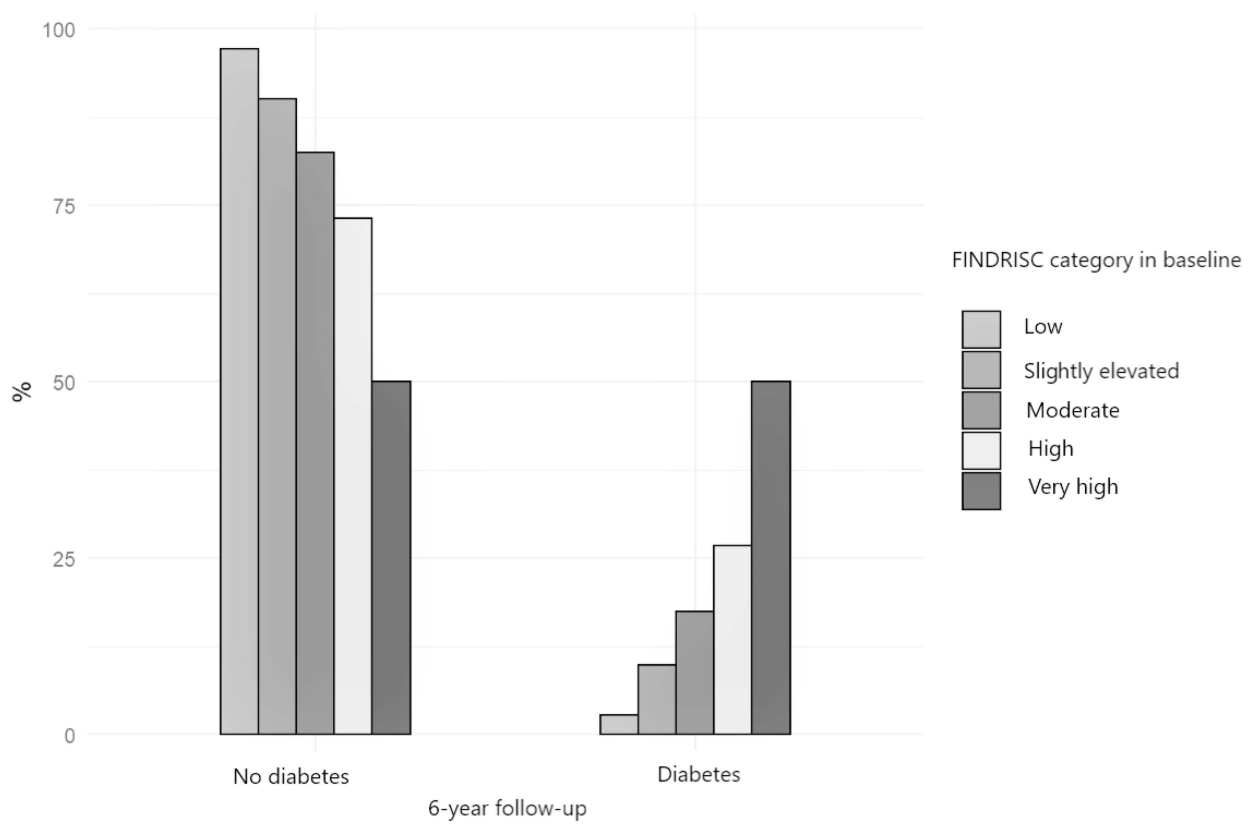

Figure I Qualitative assessment of FINDRISC score in participants with and without diabetes after 6 years of observation.

Diabetes is becoming one of the greatest challenges for public health worldwide. Accurate prognosis and early identification of people at high risk is essential to manage the epidemic of diabetes. The FINDRISC questionnaire proved to be a useful and easy tool in screening people at high risk of deterioration of glucose metabolism. According to Abraham et $\mathrm{al}^{21}$ the FINDRISC score's accuracy to identify patients with diabetes was similar to metabolic syndrome's, but was much easier to perform. On the other hand, in the same study, addition of HbA1c measurement to FINDRISC questionnaire resulted in outperforming metabolic syndrome in prognostic value of progression to diabetes. In a study performed by Schwarz et $\mathrm{al}^{22}$ FINDRISC score was significantly associated with occurrence of insulin resistance and deterioration of glucose metabolism. Authors conclude that because insulin resistance usually precedes impaired glucose tolerance (IGT), FINDRISC score can be a useful tool in identification of patients in early stages of glucose metabolism impairment. ${ }^{22}$
Utility of FINDRISC in identification of diabetes was also observed in other prospective and cross-sectional studies. ${ }^{23,24}$ Average FINDRISC score in other prospective cohort studies was comparable to our results. In the HUNT Study conducted in Norway, the mean FINDRISC score equaled 8.8. In Feel4Diabetes study high income European countries tended to have higher FINDRISC values than lower income countries. ${ }^{23}$ The average FINDRISC in high income countries (Finland, Belgium) was 10.5, in high income countries under austerity measures (Spain, Greece) was 10.8 and in low income countries (Hungary, Bulgaria) was 8.35 .

In our cohort $18.4 \%$ participants received $\geq 15$ points (which reflects high and very high risk of diabetes development), in comparison to $11 \%$ observed in the HUNT Norwegian Study ${ }^{25}$ and $12.8 \%$ in Feel4Diabetes study. ${ }^{23}$ The higher percentage of participants of high risk of diabetes in our cohort can be partially explained by overrepresentation of older participants and participants with overweight and obesity.

Table 3 Risk of Diabetes Development According to Category of FINDRISC in Baseline

\begin{tabular}{|l|l|l|l|}
\hline FINDRISC & $\begin{array}{l}\text { Participants without Diabetes in } \\
\text { 6-Year-Follow-Up n(\%) }\end{array}$ & $\begin{array}{l}\text { Participants with Diabetes in } \\
\text { 6-Year-Follow-Up n(\%) }\end{array}$ & $\begin{array}{l}\text { 6-Year Incidence of } \\
\text { Diabetes (\%, 95\% Cl) }\end{array}$ \\
\hline Low (0-6) & $274(97.2)$ & $8(2.8)$ & $0.03(0.01-0.05)$ \\
Slightly elevated (7-II) & $382(90.1)$ & $42(9.9)$ & $0.09(0.07-0.13)$ \\
Moderate (I2-I4) & I5I (82.5) & $32(17.5)$ & $0.17(0.13-0.23)$ \\
High (I5-20) & I3I (73.2) & $48(26.8)$ & $0.26(0.21-0.34)$ \\
Very high (>20) & II (50.0) & II (50.0) & $0.5(0.31-0.69)$ \\
\hline
\end{tabular}


Interestingly, $17.5 \%$ of participants who were ascertained to "moderate risk" according to FINDRISC, developed diabetes after 6 years. Surprisingly high percentage of participants developing diabetes in this category indicate that despite "moderate" risk, those participants should also implement preventive measures. Similarly, in the study by Jølle et al, highest cumulative risk of diabetes development after 10 years was observed in participants who received 7-9 points in FINDRISC score. ${ }^{26}$ Authors concluded that lowering the definition of elevated FINDRISC to $\geq 11$ would identify $>70 \%$ of those patients, who will develop diabetes within 10 years.

Similarly to our results, in the HUNT Norwegian study, women had higher mean value of FINDRISC than men (9.1 in women vs 8.4 in men). ${ }^{26}$ Higher values of FINDRISC in women were also observed in Greek arm of the DE-PLAN study (13.6 in women vs 12.6 in men). ${ }^{23}$ Higher values of FINDRISC suggest higher risk of diabetes, but interestingly at the 6-year follow-up diabetes was diagnosed more often in men. In our cohort, in comparison of FINDRISC variables between men and women, most of the mean values were rather comparable. The only variables with visible differences between sexes were consumption of fruits and vegetables and family history of diabetes. Diabetes among closest relatives was more common in women than men, on the other hand women more often met the requirements of consumption of fruit and vegetables. Those two variables are not equally important in FIDRISC, occurrence of diabetes in parents/siblings adds 5 points, whereas low consumption of fruit and vegetables adds 1 point. ${ }^{7}$ It cannot be ruled out that the interpretation of diabetes risk according to FINDRISC may be different in men and women. This issue requires further research.

In our study, participants living in rural area had significantly higher FINDRISC than those living in urban area. According to meta-analysis performed by Zabetian et al, globally 1 in every 15 persons living in rural areas is affected by diabetes. $^{27}$ Over the observed period (1990-2010), diabetes prevalence in rural areas in high income countries increased twofold, whereas in low income countries increased fourfold. ${ }^{27}$ Significant disparities in diabetes prevalence were also observed in other studies. In a cross-sectional study conducted in population from USA, participants living in rural areas had significantly higher risk of diabetes and coronary heart disease, presumably resulting from higher prevalence of obesity, tobacco smoking and poverty than in urban areas. ${ }^{28}$ Diabetes morbidity and mortality has been previously associated with lower socioeconomic status and level of education. ${ }^{29,30}$ A deprivation index of the area of residence has been associated with higher mortality and hospitalization due to diabetes, especially in women. ${ }^{31}$

Some limitations of our study must be considered. Following global PURE study protocol, in our cohort glucose parameters were ascertained only in the fasting state. Diabetes was ascertained on the basis of medical history and self-reported diabetes medication and/or fasting glucose level exceeding $126 \mathrm{mg} / \mathrm{dL}$. There is no glucose tolerance test (OGTT) performed and therefore we had only data regarding IFG, but no Impaired Glucose Tolerance (IGT). Moreover, considering limitations of our retrospective data we had to adjust our calculations of FINDRISC score (diet, history of hyperglycemia and family history of diabetes), which might have caused underestimations of the score in some participants. Having said that, we have not excluded those parameters, considering available data reliable enough in assessment of diabetes risk. Our study is also a cohort study, with overrepresentation of middle-aged and elderly participants and overrepresentation of women in comparison to general population and the results should be therefore interpreted with caution. On the other hand, the strength of our study is its prospective design, large number of participants and consistent protocol throughout the longitudinal observation. In this study, the FINDRISC score was predictive of diabetes development in 6 years of observation. Since it is not an observation period for which the FINDRISC tool has been validated for, the analysis has to be repeated in the next follow-ups in the PURE Poland cohort study.

\section{Conclusions}

Higher FINDRISC score at the baseline was associated with higher risk of diabetes development during 6 years of observation, which makes it a good predictor of diabetes development.

\section{Author Contributions}

All authors made a significant contribution to the work reported, whether that is in the conception, study design, execution, acquisition of data, analysis and interpretation, or in all these areas; took part in drafting, revising or critically reviewing the article; gave final approval of the version to be published; have agreed on the journal to which the article has been submitted; and agree to be accountable for all aspects of the work.

\section{Funding}

The main PURE study and its components are funded by the Population Health Research Institute, the Canadian Institutes 
of Health Research, Heart and Stroke Foundation of Ontario and through unrestricted grants from several pharmaceutical companies, Poland cohort study is funded by Polish Ministry of Science and Higher Education (grant no. 290/W-PURE/ 2008/0). Additionally, presented research results, were conducted within the framework of the topic: "Analiza rozpowszechnienia czynników ryzyka chorób cywilizacyjnych $\mathrm{w}$ populacji osób dorosłych oraz dzieci w wieku szkolnym z uwzględnieniem miejsca zamieszkania (miasto-wies)" (eng., Study of prevalence of risk factors of noncommunicable diseases in adults and school-aged children considering the place of residence (urban-rural)") as per records in SIMPLE system number SUB. C. 300.20.033. We declare that funding body had no role in the design of the study, collection, analysis, or interpretation of data and no role in writing the manuscript.

\section{Disclosure}

The authors report no conflicts of interest in this work.

\section{References}

1. International Diabetes Federation. IDF Diabetes Atlas. 9th ed.; 2019. Available from: https://diabetesatlas.org/en/resources/. Accessed December 20, 2019.

2. Kalbarczyk WP Cukrzyca - Gdzie Jesteśmy, Dokąd Zmierzamy. Raport Instytutu Ochrony Zdrowia; 2018. Available from: www.rpp. gov.pl Accessed December 12, 2018. .

3. Rawshani A, Rawshani A, Franzén S, et al. Mortality and cardiovascular disease in Type 1 and Type 2 diabetes. $N$ Engl J Med. 2017;376 (15):1407-1418. doi:10.1056/NEJMoa1608664

4. Su Y, Liu X-M, Sun Y-M, Wang -Y-Y, Luan Y, Wu Y. Endothelial dysfunction in impaired fasting glycemia, impaired glucose tolerance, and Type 2 diabetes mellitus. Am J Cardiol. 2008;102:497-498. doi:10.1016/j.amjcard.2008.03.087

5. Lindst JO, Louheranta A, Mannelin M, et al. The Finnish Diabetes Prevention Study (DPS) lifestyle intervention and 3-year results on diet and physical activity. Diabetes Care. 2003;26(12):3230-3236.

6. Knowler WC, Barrett-Connor E, Fowler SE, et al. Reduction in the incidence of Type 2 diabetes with lifestyle intervention or metformin. N Engl J Med. 2002;346(6):393-403. doi:10.1056/NEJMoa012512

7. Lindstrom J, Tuomilehto J. The Diabetes Risk Score: a practical tool to predict type 2 diabetes risk. Diabetes Care. 2003;26:725-731.

8. Kengne AP, Beulens JWJ, Peelen LM, et al. Non-invasive risk scores for prediction of type 2 diabetes (EPIC-InterAct): a validation of existing models. Lancet Diabetes Endocrinol. 2014;2(1):19-29. doi:10.1016/S2213-8587(13)70103-7

9. Janghorbani M, Adineh H, Amini M. Finnish Diabetes Risk Score to predict type 2 diabetes in the Isfahan diabetes prevention study. Diabetes Res Clin Pract. 2013;102(3):202-209. doi:10.1016/J. DIABRES.2013.10.018

10. Tan C, Sasagawa Y, Kamo K, et al. Evaluation of the Japanese Metabolic Syndrome Risk Score (JAMRISC): a newly developed questionnaire used as a screening tool for diagnosing metabolic syndrome and insulin resistance in Japan. Environ Health Prev Med. 2016;21(6):470. doi:10.1007/S12199-016-0568-5

11. Kulkarni M, Foraker RE, McNeill AM, et al. Evaluation of the modified FINDRISC diabetes score to identify individuals at high risk for diabetes among middle-aged white and black ARIC study participants. Diabetes Obes Metab. 2017;19(9):1260. doi:10.1111/DOM.12949
12. Zatońska K, Basiak-Rasała A, Rózańska D, et al. Changes in diabetes prevalence and corresponding risk factors-findings from 3- A nd 6-year follow-up of PURE Poland cohort study. BMC Public Health. 2020;20:843. doi:10.1186/s12889-020-08970-5

13. Teo K, Chow CK, Vaz M, Rangarajan S, Yusuf S; PURE Investigators-Writing Group. The Prospective Urban Rural Epidemiology (PURE) study: examining the impact of societal influences on chronic noncommunicable diseases in low-, middle-, and high-income countries. Am Heart J. 2009;158(1):1-7.e1. doi:10.1016/j.ahj.2009.04.019

14. Araszkiewicz A, Bandurska-Stankiewicz E, Budzyński A, et al. 2019 guidelines on the management of diabetic patients. A position of diabetes Poland. Clin Diabetol. 2019;8(1):1-95.

15. Craig CL, Marshall AL, Sjöström M, et al. International physical activity questionnaire: 12-Country reliability and validity. Med Sci Sports Exerc. 2003;35(8):1381-1395. doi:10.1249/01.MSS.0000078924.61453.FB

16. Dehghan M, Ilow R, Zatonska K, et al. Development, reproducibility and validity of the food frequency questionnaire in the Poland arm of the Prospective Urban and Rural Epidemiological (PURE) study. J Hum Nutr Diet. 2012;25(3):225-232. doi:10.1111/j.1365-277X.2012.01240.x

17. United States Department of Agriculture and Department of Health and Human Services. 2015 - 2020 Dietary Guidelines for Americans; 2015. doi:10.1097/NT.0b013e31826c50af

18. Jarosz M. Nutritional Guidelines for Polish Population (Normy Żywienia Dla Populacji Polski). Instytut Żywności i Żywienia; 2017. http://zywnosc.com.pl/wp-content/uploads/2017/12/normyzywienia-dla-populacji-polski-2017-1.pdf. Accessed March 2, 2018.

19. Rydén L, Grant PJ, Anker SD, et al. ESC guidelines on diabetes, pre-diabetes, and cardiovascular diseases developed in collaboration with the EASD. Eur Heart J. 2013;34(39):3035-3087. doi:10.1093/ eurheartj/eht108

20. R Core Team. R: A language and environment for statistical computing. Vienna, Austria: R Foundation for Statistical Computing; 2019. Available from: https://www.r-project.org/. Accessed June 25, 2019.

21. Meijnikman AS, De Block CEM, Verrijken A, Mertens I, Van Gaal LF. Predicting type 2 diabetes mellitus: a comparison between the FINDRISC score and the metabolic syndrome. Diabetol Metab Syndr. 2018;10:1. doi:10.1186/s13098-018-0310-0

22. Schwarz PEH, Li J, Reimann M, et al. The Finnish diabetes risk score is associated with insulin resistance and progression towards type 2 diabetes. J Clin Endocrinol Metab. 2009;94(3):920-926. doi:10.1210/jc.2007-2427

23. Mavrogianni C, Lambrinou CP, Androutsos O, et al. Evaluation of the Finnish Diabetes Risk Score as a screening tool for undiagnosed type 2 diabetes and dysglycaemia among early middle-aged adults in a large-scale European cohort. The Feel 4 Diabetes-study. Diabetes Res Clin Pract. 2019;150:99-110. doi:10.1016/j.diabres.2019.02.017

24. Tankova T, Chakarova N, Atanassova I, Dakovska L. Evaluation of the Finnish Diabetes Risk Score as a screening tool for impaired fasting glucose, impaired glucose tolerance and undetected diabetes. Diabetes Res Clin Pract. 2011;92(1):46-52. doi:10.1016/j.diabres.2010.12.020

25. Jølle A, Midthjell K, Holmen J, et al. Impact of sex and age on the performance of FINDRISC: the HUNT study in Norway. BMJ Open Diabetes Res Care. 2016;4:1. doi:10.1136/bmjdrc-2016-000217

26. Jølle A, Midthjell K, Holmen J, et al. Validity of the FINDRISC as a prediction tool for diabetes in a contemporary Norwegian population: a 10-year follow-up of the HUNT study. BMJ Open Diabetes Res Care. 2019;7:1. doi:10.1136/bmjdrc-2019-000769

27. Hwang CK, Han PV, Zabetian A, Ali MK, Venkat Narayan KM. Rural diabetes prevalence quintuples over twenty-five years in lowand middle-income countries: a systematic review and meta-analysis. Diabetes Res Clin Pract. 2012;96(3):271-285. doi:10.1016/j. diabres.2011.12.001

28. O'Connor A, Wellenius G. Rural-urban disparities in the prevalence of diabetes and coronary heart disease. Public Health. 2012;126 (10):813-820. doi:10.1016/j.puhe.2012.05.029 
29. Espelt A, Borrell C, Roskam AJ, et al. Socioeconomic inequalities in diabetes mellitus across Europe at the beginning of the 21st century. Diabetologia. 2008;51(11):1971-1979. doi:10.1007/s00125-008-1146-1

30. Agardh E, Allebeck P, Hallqvist J, Moradi T, Sidorchuk A. Type 2 diabetes incidence and socio-economic position: a systematic review and meta-analysis. Int J Epidemiol. 2011;40(3):804-818. doi:10.1093/ ije/dyr029
31. Fano V, Pezzotti P, Gnavi R, et al. The role of socio-economic factors on prevalence and health outcomes of persons with diabetes in Rome, Italy. Eur J Public Health. 2013;23(6):991-997. doi:10.1093/eurpub/cks168

\section{Publish your work in this journal}

Vascular Health and Risk Management is an international, peerreviewed journal of therapeutics and risk management, focusing on concise rapid reporting of clinical studies on the processes involved in the maintenance of vascular health; the monitoring, prevention and treatment of vascular disease and its sequelae; and the involvement of metabolic disorders, particularly diabetes. This journal is indexed on PubMed Central and MedLine. The manuscript management system is completely online and includes a very quick and fair peerreview system, which is all easy to use. Visit http://www.dovepress. com/testimonials.php to read real quotes from published authors. 